\title{
Thermal Inertia: Towards An Energy Conservation Room Management System
}

\author{
Dawei Pan ${ }^{\dagger}$, Yi Yuan*, Dan Wang*, Xiaohua Xu ${ }^{\ddagger}$, Yu Peng ${ }^{\dagger}$, Xiyuan Peng ${ }^{\dagger}$, Peng-Jun Wan ${ }^{\ddagger}$ \\ *The Hong Kong Polytechnic University, ${ }^{\dagger}$ Harbin Institute of Technology, ${ }^{\ddagger}$ Illinois Institute of Technology
}

\begin{abstract}
We are in an age where people are paying increasing attention to energy conservation around the world. The heating and air-conditioning systems of buildings introduce one of the largest chunk of energy expenses. In this paper, we make a key observation that after a meeting or a class ends in a room, the indoor temperature will not immediately increase to the outdoor temperature. We call this phenomenon Thermal Inertia. Thus, if we arrange subsequent meetings in the same room; than a room that has not been used for some time, we can take advantage of such un-dissipated cool or heated air and conserve energy.

We develop a green room management system with three main components. First, it has a wireless sensor network to collect indoor, outdoor temperature and electricity expenses of the air-conditioning devices. Second, we build an energytemperature correlation model for the energy expenses and the corresponding room temperature. Third, we develop room scheduling algorithms. Our system is validated with real deployment of a sensor network for data collection and thermodynamics model calibration. We conduct a comprehensive evaluation with synthetic room and meeting configurations. We observe a $\mathbf{3 0 \%}$ energy saving as compared with the current schedules.
\end{abstract}

\section{INTRODUCTION}

There is a huge interest in building a green world recently. The key focus is energy conservation and energy efficiency. Computer scientists are actively contributing our effort in two directions, 1) improve energy efficiency of computing systems, and 2) apply computing systems (e.g., sensor networks) for energy conservation in broader disciplines.

For the first category, many studies are working on energy efficiency for data centers [5][10][11][13], a top energy consumer among all computing devices. While the energy expenses of computing industry are increasing fast in recen$t$ years, the largest portion of energy consumption is still dominated by such areas as commercial buildings, residential usage, transportation, manufactory industry [14]. Especially, for regions where the Industrial sector is small, the electricity consumption by commercial buildings can be more dominating; for example in Hong Kong, 65\% of electricity in 2008 goes to the commercial sector [3].

The heating and air conditioning of commercial buildings has the largest chunk in energy expenses. In 2008 the Office Segment of Hong Kong, 54\% electricity goes to space conditioning (i.e., air-conditioning), $14 \%$ goes to lighting, $13 \%$ goes to office equipments such as computers [3]. Monitoring the conditions of the buildings and efficient utilization of heating, ventilation, and air conditioning (HVAC) have been a long time topic[6][8][9]; and advanced commercial buildings can automatically turn off lights and HVAC systems of rooms when humans are not in presence. Nevertheless, we notice that even if the heating or air-conditioning of a room is turned off, the heat or the cool air will not immediately dissipate. We call this phenomenon Thermal Inertia. We consider the un-dissipated cool or heated air a valuable resource that can be utilized, so that future usage of this room can take this advantage without re-heating or re-cooling the room.

Based on this observation, we develop an energy conservation room management system, such that the allocation of the rooms of a building (or classrooms in campus) is based not only on a schedule (e.g., meeting time, room capacity), but also on the existing heating or air-conditioning conditions of the rooms. In the rest of the paper, we will only use airconditioning as an example to ease our presentation.

Clearly, our room management system falls into an optimization problem. It is not straightforward, however to know how much energy will be saved if a room is scheduled. As an example, consider a recommended office temperature to be $26^{\circ} \mathrm{C}\left(79^{\circ} \mathrm{F}\right)$. Assume a room was used 20 minutes ago, and its current temperature is $29^{\circ} \mathrm{C}\left(84^{\circ} \mathrm{F}\right)$. The outdoor temperature is $37^{\circ} \mathrm{C}\left(99^{\circ} \mathrm{F}\right)$. If we schedule a meeting 5 minutes later in this room, how much electricity is needed to re-cool it to the targeted temperature $26^{\circ} \mathrm{C}\left(79^{\circ} \mathrm{F}\right)$ ?

This is affected by such factors as the room specifics (size, wall materials, etc), indoor and outdoor temperature, the targeted temperature etc. A key difficulty is to build a correlation among these factors. The more accurate this correlation model is, the better the scheduling algorithm we can run on top of it. Building this model does not solely fall into the computer science domain. Advanced thermodynamics theories may be needed. We believe that in the sensor network research today, it is very common that cross-discipline understandings are required; for example, it is shown that knowledge on sensor placement quality in the sense of civil engineering can make the structural health monitoring system built by computer scientists more plausible [4]. A careful management on the degree of understanding on different disciplines is very important. In our work, we choose to apply rudimental thermodynamics theory to build an initial energy-temperature model. We then use sensor data to calibrate this model. We validate the effectiveness of such design by a real experiment.

Another difficulty is that we do not have off-the-shelf components for our sensor network. We thus extend Imote 2 to an electricity-meter in order to record electricity usage of airconditioners. On top of these, we develop room scheduling algorithms. We first develop an optimal algorithm for a special case where all rooms are equal. For the general case, we develop two efficient heuristics. 
Besides a real world system deployment for model validation and data collection, we evaluate our system with comprehensive simulations with synthetic room configurations and meeting schedules. We observe that we can save $30 \%$ of electricity as compared to the synthetic data.

\section{Room Management System: An Overview}

We discuss some high level system choices. As a first work, we confine our study that given the schedules, how the classes/meetings should be arranged. We leave a detailed investigation of online room management as future work.

To accurately schedule rooms and maximally conserve energy, an important part of our system is that we need to build an energy-temperature correlation model so that the room scheduling algorithm can run on top of it. More specifically, we need a function such that given the current temperature and room environment configurations, the energy to be consumed to achieve the target temperature. There are two extreme ways for building such model. First we can apply advanced thermodynamics theories and material sciences to explicitly compute such function. Second, we can build a database with entries of the environment parameters (e.g., indoor temperature, outdoor temperature, and targeted temperature) and the corresponding energy consumptions. In the room scheduling algorithm, whenever an estimation on the energy expenses is needed, an entry in this database that has the most similar environmental configuration can be extracted.

The first choice falls into the expertise of Building and Service Engineering. We have consulted experts of BSE from both academia and industry. While there are sophisticated tools such as EnergyPlus [1], they admit that it is difficult to build a model purely from theory. For the second choice, to build the correlation database, a sensor network can be deployed to collect such data as temperature and energy expenses. The accuracy depends on the granularity of the data collection. The more samples the database has, the more accurate to find the energy expenses with a similar environmental configuration. After some studies on physical laws on heat conduction and some field experimental validation, our choice finally falls into a mixture of the two extremes. We use an initial model following rudimental Fourier's law of heat conduction. In this model, some parameters are difficult to compute from theory. These parameters are invariants, however, e.g., only affected by the materials of the room. Thus we inversely calibrate the parameters of this model using the data collected by a sensor network. The high-level framework of our system is in Fig. 1.

We also want to clarify that in this paper, we use electricity expenses as our optimization objective. For end-users, having their electricity bills cut directly means money saving.

\section{Sensor Network Design}

For a building, or a campus, there are multiple rooms. For each room, we need to build an energy-temperature correlation model (detail in Section IV) to be used for the scheduling algorithm (details in Section V). As such, a sensor network should be deployed in each room. In this sensor network,

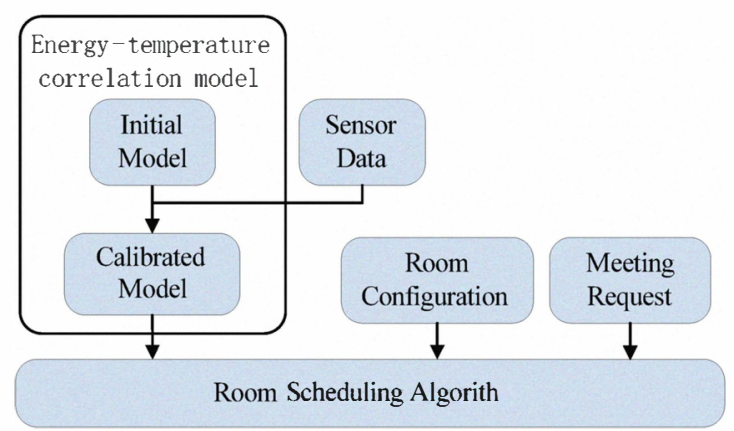

Fig. 1: The framework of the room management system.

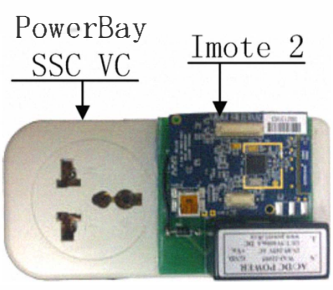

Fig. 2: Electricity-Meter

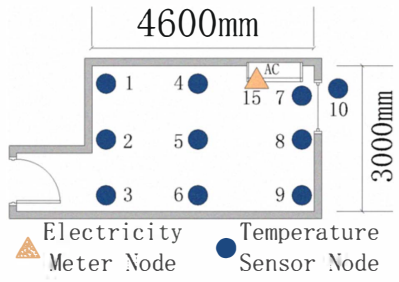

Fig. 3: Experiment Environment there should be a sensor to record electricity usage to airconditioning the room. We also need to record the temperature. As the temperature in different locations of the room may not be uniform, a set of temperature sensors is suggested. We would like to comment that the sensor network is only used for the construction of the energy-temperature correlation model for each room. After the model is built, we can predict the energy consumption using the model. Since the sensor network needed in each room is the same, in practice, we can deploy a sensor network and build the energy-temperature correlation model room-by-room.

\section{A. Design of an Electricity-meter}

Our system needs to estimate the energy consumption for air-conditioning the room to a targeted temperature. We extend Imote2 with a PowerBay SSC VC to record electricity current (see Fig. 2). PowerBay SSC VC also becomes a power supply to Imote2. In operation, PowerBay SSC VC will record the power (in Watt) and such data will be digitized and output to Imote2. The data can then be transmitted out by Imote2.

\section{B. Development of Sensor Network}

We implement our sensor system in TinyOS, and use Collect Tree Protocol (CTP) [2] for data routing among sensor nodes. The temperature and electricity data are sent to a base station node attached to a laptop. The lifetime of our sensor system is determined by TelosB nodes if they use battery power. In practice, every node gets the temperature and transmits 32 bytes every 10 seconds; The projected lifetime of our sensor network can thus reach 2000 hours. We find that this is far enough for us to collect data and calibrate the energytemperature correlation model.

\section{Design of Energy-Temperature Correlation MODEL AND EXPERIMENTAL VALIDATION}

In this section, we develop a model where the electricity is a function of (a room, current indoor/outdoor temperature, 
targeted temperature). Our idea is based on the observation that thermodynamic factors of a room are invariants. They are determined by their physical materials and do not change (or change ignorably) with outside factors. Therefore, for each real-world room, we can build a virtual perfect room to mimic it. For this virtual perfect room, we build an energy-temperature correlation model using Fourier's law of heat conduction with the set of invariants undetermined. To compute these invariants, we collect a set of electricity and temperature data by our sensor network. We then inversely derive these invariants. After fitting these invariants back to the model, we can compute electricity usage in the room given any indoor/outdoor temperature and targeted temperature.

\section{A. Energy-Temperature Correlation Model}

We use a virtual perfect room where 1) The room space is enclosed, i.e., no air exchange with other spaces; 2) All walls, ceiling and floor are made of materials with the same thermal conductivity and have identical thickness; 3) All outside temperature of the room is same and is constant. We also assume 1) the electrical power $P$ of the air conditioner is constant when it is in operation, and is zero if it stops; and 2 ) the electricity-energy transformation rate $r$ is a constant; this indicates the effective energy $P_{e}$ injected into a room per second when an air-conditioner is in operation is constant.

Let $T$ be the indoor temperature. Let $T_{o}$ be the temperature outside the room. Let $Q$ be the heat transfer rate from outdoor to the room. Let $k$ be the thermal conductivity of the material. Let $A$ be the total area of the six walls. Let $L$ be the thickness of a material. According to Fourier's law [7], we have $Q=$ $\frac{k A}{L}\left(T_{o}-T\right)$. Let $m$ be the mass of the air of the room. Let $C$ be the heat capacity of the air of the room. The temperature changing rate $\frac{d T}{d t}$ of the room [12] is $\frac{d T}{d t}=\frac{Q+P_{e}}{m C}$. Let $\lambda=$ $\frac{k A}{L}$. We say $\lambda$ as the conductivity of this specific room. We obtain the following function for indoor temperature change:

$$
T(t)=T_{o}+P_{e} \times \frac{1}{\lambda}+C_{0} e^{-\frac{\lambda}{m C} t}
$$

Here $C_{0}$ is an initialization parameter determined by $T(0)$, the temperature at time 0 :

$$
C_{0}= \begin{cases}T(0)-T_{o} ; & \text { air-conditioner not in operation } \\ T(0)-T_{o}-\frac{P_{e}}{\lambda} ; & \text { air-conditioner in operation }\end{cases}
$$

The energy-temperature correlation model is Eq.1 and Eq.2. We consider $\lambda$ as an invariant, because it is related to the physical properties of the materials. Therefore, we calibrate this parameter by sensor data. We also calibrate $T_{o}$. We emphasize that $T_{o}$ is artificial that approximates the overall outdoor situation of all walls. Though one wall may have a bigger change in outdoor temperature, $T_{o}$ does not change abruptly. We will show that this is true in our experiments.

We use the sensor data to inversely compute the invariants $\lambda, r$ and semi-invariant $T_{o}$. We use $\hat{\lambda}, \hat{T}_{o}$ and $\hat{r}$ to denote them. Then we fit $\hat{\lambda}, \hat{T}_{o}$ and $\hat{r}$ back into Eq.1 and Eq. 2 to fulfill our model.

The operation of a room can be cut into three periods: 1) the vacancy period (VP); 2) the re-cooling period (RP); and 3) the maintaining period (MP). The energy-temperature function

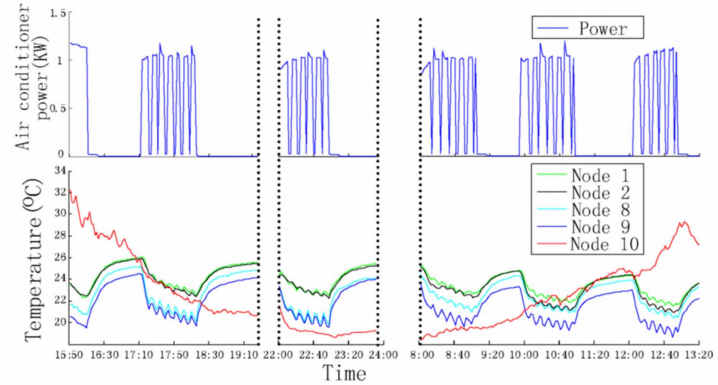

Fig. 4: Experiment Results

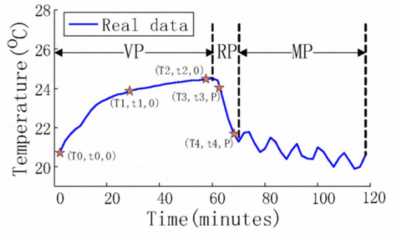

Fig. 5: Data selection for IndividualCal()

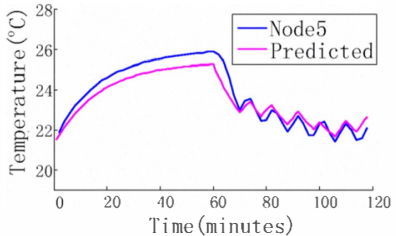

Fig. 6: Predicted $T$ vs. temperature data of Node 5. of VP and RP are different (see the two phases of Eq.1 and Eq.2). MP is a combination of short periods of VP and RP.

Through the sensor network, we will collect a temperature sequence for each sensor node $i$ and an electricity sequence. For each of these sequences, we identify periods of VPs, RPs and MPs. For each node $i$, we then apply Algorithm IndividualCal() to calibrate $\hat{\lambda}_{i}, \hat{T}_{o i}$ and $\hat{r}_{i}$. The basic idea is we select three points in VP to calculate $\hat{\lambda}_{i}, \hat{T}_{o i}$ (See Fig.5); then select two points in RP and put the calculated $\hat{\lambda}_{i}, \hat{T}_{o i}$ into the two equations to calculate $\hat{r}_{i}$. As $\hat{\lambda}_{i}, \hat{T}_{o i}$ and $\hat{r}_{i}$ computed from each sensor $i$ are not fully equal. We apply Algorithm ModelCal() to get the final $\hat{\lambda}, \hat{T}_{o}$ and $\hat{r}$. The basic idea is to remove outliers by setting an upper error bound $\varepsilon$ and compute a weighted average of all $\hat{\lambda}_{i}, \hat{T}_{o i}$ and $\hat{r}_{i}$.

\section{B. Experiment Validation}

We conduct a real experiment to validate our model. Our experiment was conducted in a hotel room in Shenzhen, China. The configuration of the room and sensor network is shown in Fig. 3. There were ten sensors to collect temperature and an electricity-meter connected to the air-conditioner. Our experiment lasted one day from March 2nd to 3rd 2011. We periodically turned on and off the air-conditioner(AC). The result is shown in Fig. 4. The bottom part of Fig. 4 shows the temperature of four indoor sensors and the outdoor sensor. The upper part of Fig. 4 shows the corresponding output power level of the AC. Fig. 4 indicates the weak connection between the outdoor temperature (No. 10) and the indoor temperature. After getting $\left(\hat{\lambda}, \hat{T}_{o}, \hat{r}\right)=(58.72,25.38,-0.32)$, we fit them back to our model. We draw a predicted temperature curve in Fig. 6 by applying the same initial temperature, the same energy sequence. Compared with the real temperature sequence of Node 5. We see that the predicted temperature is fairly close to the real temperature sequence. Thus we conclude that our model can be used to estimate future room electricity usage.

\section{Room Scheduling Algorithm}

With the energy-temperature correlation model, we are prepared to develop the room scheduling algorithm. We have 
searched existing room scheduling algorithm in literature. To the best of our knowledge, we did not find any standard algorithm. We believe ad-hoc scheduling is used because of two reasons: 1) the number of rooms is not always tight, 2) there is no optimization objective, only to fit the meetings in. As such, advanced algorithms might not be necessary.

We formally state the problem. Given a set $\mathcal{R}$ of $n$ rooms and a set $\mathcal{M}$ of $m$ meetings to be scheduled. A meeting $M_{i} \in \mathcal{M}$ is associated with a time interval $\left(b_{i}, e_{i}\right)$ and a target temperature $T_{t i}$, where $b_{i}, e_{i}$ represent the start time and the end time of the meeting respectively. Each meeting $M_{i}$ has a capacity requirement $c_{i}$. Each room $R_{j} \in \mathcal{R}$ has a capacity $C_{j}$. For $R_{j}$ that can hold $M_{i}$, we must have $c_{i}<C_{j}$. Every room $R_{j}$ is associated with a function $E_{j}\left(T_{t}, t\right)$ showing the energy needed to maintain the target temperature $T_{t}$ for $t$ and a function $R E_{j}\left(T_{t}, t\right)$ showing the energy needed to re-cool the room to $T_{t}$ where last meeting has ended for $t . E_{j}\left(T_{t}, t\right)$ and $R E_{j}\left(T_{t}, t\right)$ can be computed by our energy-temperature correlation model. We want to find a schedule $\mathcal{S}$ consisting a set of time intervals, one for each meeting. The objective is to reduce the total energy of $\mathcal{S}$.

We first develop an optimal algorithm when the rooms are uniform. For the general problem with non-uniform rooms, we develop two fast heuristics for different scenarios.

\section{A. Rooms with Uniform Capacity}

Our algorithm Energy-Aware Room Scheduling (Uniform), Energy-RS(Uniform) for short, is a greedy-based algorithm. We sort the meetings in ascending order based on their starting times. We then group the meetings with the same starting time. Our algorithm performs in iterations and in each iteration, we handle a group of meetings with the current earliest starting time. We allocate these meetings to the rooms that have ending times that are closest these meetings. Due to page limits, the pseudo-code of our algorithm is in [15].

Theorem 1: The total energy consumption by Algorithm Energy-RS(Uniform) is minimum.

Proof: Due to page limitation, please refer to [15]

Theorem 2: The total number of rooms scheduled by Algorithm Energy-RS(Uniform) is minimum.

Proof: Due to page limitation, please refer [15].

Existing ad-hoc meeting scheduling algorithms usually do not bother if using more rooms. This theorem indicates that Algorithm Energy-RS (Uniform) will select the smallest number of rooms. This is useful for the general algorithm with non-uniform rooms; since we try not to schedule meetings with small capacity requirements into oversized rooms.

\section{B. Rooms with Non-Uniform Capacity}

1) Energy-RS(): We use Algorithm Eenergy-RS (Uniform) as a building block to develop algorithm Energy-Aware Room Schedule (Energy-RS()). We outline our basic idea. Assume the number of different capacities of all rooms is $g$. We classify the rooms into different groups $\mathcal{R G}_{1}, \mathcal{R G}_{2}, \ldots, \mathcal{R G}_{g}$ according to their capacity. Let $G C_{k}$ be the room capacity of $\mathcal{R} \mathcal{G}_{k}$. We have $\forall R_{j} \in \mathcal{R} \mathcal{G}_{k}, C_{j}=G C_{k}$. Assume

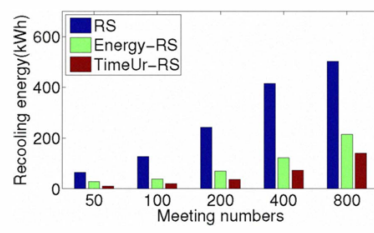

(a)

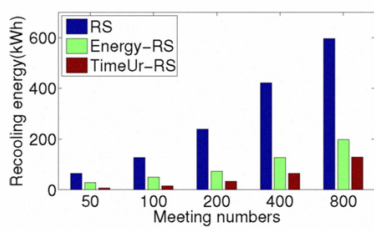

(b)
Fig. 7: Total energy expense for re-cooling the rooms as against to the number of meetings; rooms with uniform capacity. (a) meeting length: option $\mathcal{O}_{1}$, (b)

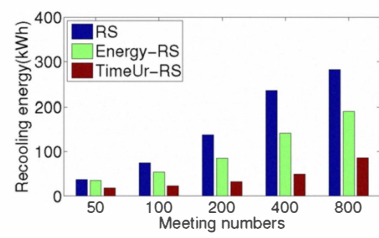

(a)

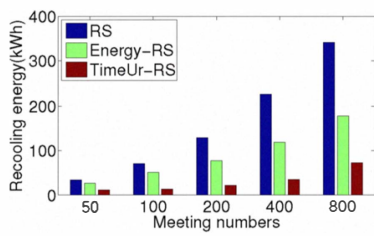

(b)
Fig. 8: Total energy expense for re-cooling the rooms as against to the number of meetings; rooms with non-uniform capacity. (a) meeting length: option $\mathcal{O}_{1}$, (b) meeting length: option $\mathrm{O}_{2}$

$\mathcal{R G}_{1}, \mathcal{R G}_{2}, \ldots, \mathcal{R G}_{g}$ is sorted in ascending order according to their capacity $G C_{k}$. We classify the meeting into different groups $\mathcal{M G}_{1}, \mathcal{M G}_{2}, \ldots, \mathcal{M G}_{g}$ according to the capacity requirements of the meetings. For a meeting $M_{i}$ with a capacity requirement $c_{i}$, it is grouped into $\mathcal{M \mathcal { G } _ { k }}$ where $G C_{k-1}<c_{i} \leq$ $G C_{k}$. As an example, assume the room capacities of all rooms are $20,40,60$. The meeting requirements are $17,18,34$. We thus classify the meetings with capacity requirements of 17 and 18 into the group of 20 and the meeting with capacity requirement of 34 into the group of 40 .

We schedule meetings of $\mathcal{M \mathcal { G } _ { k }}$ into room group $\mathcal{R \mathcal { G } _ { k }}$ in ascending order. For each group pair $\left(\mathcal{M G}_{k}, \mathcal{R G}_{k}\right)$, we apply Algorithm Energy-RS (Uniform). From Theorem 2, we know that Algorithm Energy-RS (Uniform) uses the smallest number of rooms. Thus, the chance that a meeting with small capacity requirement is pushed into an oversized room is minimized. The complexity of Algorithm Energy-RS() is $n m$.

2) TimeUr-RS(): In our framework, each meeting has a capacity requirement and a meeting time requirement. This is the case for many scenarios. For some cases, however, the meeting time can be determined by the room scheduling system. We conjecture this is a general case since there is no optimization goal for many meeting schedules; only to fit all the meetings in without meeting-meeting, room-room conflict.

We propose a simple greedy-based algorithm which allows reassignment of meeting times, we call Time Unrestricted Energy Aware Room Scheduling (TimeUr-RS()). TimeUr-RS() is greedy by sorting meeting capacities in descending order and then fitting into the rooms. This algorithm can be used to provide suggestions for the decision makers, in case there is no compulsory reason to have strict meeting times. In our simulation, TimeUr-RS() is used as a performance comparison.

\section{Vi. Performance Evaluation}

\section{A. Simulation setup}

We evaluate our system in a set of synthetic room arrangements we generate semi-randomly. We consider rooms with 
uniform capacity and non-uniform capacity separately.

For the uniform case, the default room capacity is 100 seats and the total number of rooms is 150 . The meeting times are randomly generated in range [8:00, 22:00]. The lengths of the meetings are randomly chosen from two groups of options, $\mathcal{O}_{1}=[1,1.5,2,2.5,3], \mathcal{O}_{2}=[1,2,3]$. For the non-uniform capacity case, we have eight different types of rooms.

The numbers of different types of rooms follow a poisson distribution with a mean of 3 . The capacity requirement for the meetings follows a poisson distribution with a mean of 3 .

The default values of our simulation are $\hat{T}_{o}=25^{\circ} C, \hat{r}=$ -0.32 for all rooms. We set $T_{t}=20^{\circ} \mathrm{C}$ for all meetings.

We compare our algorithm with an ad-hoc room scheduling algorithms (denoted as $R S$ ) that can satisfy the meeting time and room capacity requirements.

We choose our primary performance metric as the total energy needed to re-cool the rooms to the target temperature for all rooms and all meetings. Note that we exclude the energy needed during the classes, which we cannot conserve. This metric is stable for all room scheduling algorithms.

\section{B. Simulation results}

In Fig. 7, we show the total energy for re-cooling the rooms for different algorithms. In Fig. 7 (a), we see that the re-cooling energy needed for ad-hoc room scheduling RS is always greater than our algorithm Energy-RS and TimeUr-RS. This is not surprising as the RS only satisfies the meeting requirements. When the number of meetings increases, we can see that all three algorithms need more energy in recooling the rooms. This is because there are more meetings and more rooms to be used. RS increases much faster than our algorithms, however; as both of our algorithms have taken the energy conservation into consideration. More specifically, we can see that if there are 800 meetings to schedule, the total electricity needed by RS, Energy-RS and TimeUr-RS is 503 $\mathrm{kWh}, 214 \mathrm{kWh}$, and $141 \mathrm{kWh}$ respective. We can see that we have reduced the electricity consumption for more than half. If the meeting time is not restricted, we can make a suggestion on meeting times so as to reduce the electricity consumption to less than one third.

We then see Fig. 7 (b) where the meeting time is randomly chosen from $\mathrm{O}_{2}$. We see similar trend as that in Fig. 7(a). We also see that the less number of choices that we have in meeting time, the greater the benefit we gain. This is because if there is a smaller number of meeting length options, there is also a smaller number of small time segments that we cannot fit the meetings in due to more irregular meeting time length. On the contrary, we do not see improve for RS as its schedule is ad-hoc.

We then study the general case where rooms are of nonuniform capacity. We show the results in Fig. 8. We see that the gain of Energy-RS is smaller. This is because, in each type of room capacity, we have a much smaller number of meeting choices. If one takes a closer look at Fig. 7 (a), we can see that the best performance arrives when the number of meetings is 800 . When the number of meetings is 100 , or 50 , the gain is smaller. In our general case, we have 8 different types of rooms resulting in a smaller number of meetings in each type. Thus, the gain is smaller. We can summarize that the more meetings, the more choices; leading to more re-cooling energy needed; and a better performance of Energy-RS as compared to RS.

\section{CONCLUSION}

In this paper, we observed Thermal Inertia; that is, after a meeting ends in a room, the cool air will not immediately dissipate. We took such advantage and designed a new room management system for energy conservation. We extended sensor hardware and designed a two tier sensor network to monitor necessary information such as indoor/outdoor temperature and electricity expenses. We developed an energytemperature correlation model and validate the model with our sensor network in real-world experiment. We further developed efficient room scheduling algorithms. Comprehensive simulations verified the effectiveness of our system.

\section{ACKNOWLEDGMENT}

Dan Wang's work was supported by Hong Kong PolyU/APJ19, A-PK95, A-PL23, 4-BC01, 4-BC02, 4-BC03, and RGC/GRF PolyU 5305/08E.

Peng-Jun Wan and Xiao-Hua Xu were supported in part by the NSF grants CNS-0831939 and CNS-0916666.

\section{REFERENCES}

[1] Basic Concepts Manual - Essential Information, the US Department of Energy, USA, http://appsl.eere.energy.gov/buildings/energyplus/pdfs/gettingstarted.pdf.

[2] O. Gnawali, R. Fonseca, K. Jamieson, D. Moss and Philip Levis, "Collection Tree Protocol", in Proc. ACM SenSys'09, Berkeley, CA, Nov. 2009.

[3] Hong Kong Energy End-Use Data, 2010, The Energy Efficiency Office, Electrical and Mechanical Service Department (EMSD), Hong Kong, http://www.emsd.gov.hk/emsd/e_download/pee/HKEEUD2010.pdf

[4] B. Li, D. Wang, F. Wang, and Y. Q. Ni, "High Quality Sensor Placement for Structural Health Monitoring Systems: Refocusing on Application Demands", in Proc. IEEE INFOCOM'10, San Diego, CA, Mar. 2010.

[5] C. Liang, J. Liu, L. Luo, A. Terzis, and Feng Zhao, "RACNet: A High-Fidelity Data Center Sensing Network", in Proc. ACM SenSys'09, Berkeley, CA. Nov., 2009.

[6] K. Lin and R. Gupta, "Towards Automated Building Management through Cooperative Sensor-actuator Networks", in Proc. HotPower'09, Big Sky, MT, Oct. 2009.

[7] J. Lienhard IV and J. Lienhard V, "A heat transfer textbook,3rd edition", pp. 12-18, 2003.

[8] J. Lu, T. Sookoor, V. Srinivasan, G. Gao, B. Holben, J. Stankovic, E. Field, K. Whitehouse, "The smart thermostat: Using Occupancy sensors to save energy in homes", in Proc. ACM SenSys'10, Zurich, Switzerland, Nov. 2010.

[9] J. Lu, D. Birru, K. Whitehouse , "Using Simple Light Sensors to Achieve Smart Daylight Harvesting", in Proc. ACM BuildSys'10, Zurich, Switzerland, Nov. 2010.

[10] L. Parolini, B. Sinopoli and B. Krogh, "Reducing data center energy consumption via coordinated cooling and load management", in Proc. HotPower'08, San Diego, CA, Dec, 2008.

[11] R. Raghavendra, P. Ranganathan, V. Talwar, Z.Wang, X. Zhu, "No Power Struggles: Coordinated Multi-level Power Management for the Data Center", in Proc. ACM ASPLOS'08, Seattle, USA, Mar, 2008.

[12] H. Sauer, R. Howell, W. Coad, "Principles of heating, ventilating, and air conditioning", Section 2.4, 2001.

[13] Y. Shang, D. Li and M. Xu, "Energy-aware Routing in Data Center Network", in Proc. ACM SIGCOMM Workshop on Green Networking'10, New Delhi, India, Aug. 2010.

[14] Energy in the United States, http://en.wikipedia.org/wiki/Energy_in_the_United_States

[15] Y. Yuan, D. Pan, D. Wang, X. Xu, Y. Peng, X. Peng, P. Wan "Thermal Inertia: Towards An Energy Conservation Room Management System." Technical report and Matlab package, Jun. 2011. Available at http://www4.comp.polyu.edu.hk/ csyiyuan/projects/ECRMS.html 